\title{
An equivalence theorem for submanifolds of higher codimensions
}

\author{
by PaweŁWitowicz (Rzeszów)
}

\begin{abstract}
For a submanifold of $\mathbb{R}^{n}$ of any codimension the notion of type number is introduced. Under the assumption that the type number is greater than 1 an equivalence theorem is proved.
\end{abstract}

Introduction. The paper deals with submanifolds of $\mathbb{R}^{n}$ of codimension greater than one, equipped with affine connections. Problems concerning higher codimensions have been studied in Riemannian geometry since the 1930-ties, for example by Allendoerfer (see [1]). He formulated and proved a few theorems about the existence and equivalence of submanifolds isometrically immersed in Euclidean spaces of high dimension ([1], [6]). A key notion used in his papers was the type number of an immersion, which is a generalization of the rank of the second fundamental form.

In affine geometry there are very few results dealing with submanifolds of any codimension (see [2], [4]).

In this paper the type number of an immersion of any codimension is defined in the affine case. Using this notion we prove an equivalence theorem. More precisely, we show that two submanifolds of type number greater than one having the same affine connections and second fundamental forms are affinely equivalent.

1. The type number of an immersion. Let $f: M^{n} \rightarrow \mathbb{R}^{n+p}$ be an immersion of an $n$-dimensional manifold $M^{n}$ into $\mathbb{R}^{n+p}$ equipped with the usual flat affine connection $\widetilde{\nabla}$. If $N$ is a vector bundle over $M^{n}$ such that

$$
\forall x \in M^{n} \quad T_{f(x)} \mathbb{R}^{n+p}=f_{*}\left(T_{x} M^{n}\right) \oplus N_{x},
$$

then for all $X, Y \in \mathcal{X}\left(M^{n}\right)$ we have a decomposition

$$
\widetilde{\nabla}_{X} f_{*}(Y)=f_{*}\left(\nabla_{X} Y\right)+h(X, Y),
$$

1991 Mathematics Subject Classification: Primary 53A13; Secondary 53C40.

Key words and phrases: submanifold, affine immersion, normal bundle. 
Here $\nabla_{X} Y$ is a local section of $T M^{n}$ and $h(X, Y)$ is a local section of $N$. Throughout the paper we write $\nabla_{X} Y \in T M^{n}$ and $h(X, Y) \in N$ to mean that $\left(\nabla_{X} Y\right)(x) \in T_{x} M^{n}$ and $h_{x}(X, Y) \in N_{x}$ for every $x$ in the domain of $\widetilde{\nabla}_{X} f_{*}(Y)$. It is easy to prove that $\nabla$ is an affine connection without torsion on $M^{n}$ and $h$, called the second fundamental form on $M^{n}$, is a bilinear symmetric mapping from $T_{x} M^{n} \times T_{x} M^{n}$ into $N_{x}$ for each fixed $x \in M^{n}$.

For a local section $\xi$ of $N$ and for any $X \in \mathcal{X}\left(M^{n}\right)$ we also have a decomposition

$$
\widetilde{\nabla}_{X} \xi=-f_{*}\left(A_{\xi} X\right)+D_{X} \xi
$$

where $A_{\xi}$ and $D_{X} \xi$ are defined by $A_{\xi} X \in T M$ and $D_{X} \xi \in N . A_{\xi}$ is a $(1,1)$ tensor field on $M^{n}$, called the shape operator, and $D_{X} \xi$ defines a connection in the vector bundle $N$, called the normal connection. We also remark that the mapping $\xi \mapsto A_{\xi}$ is linear over $\mathcal{C}^{\infty}\left(M^{n}\right)$. For fixed $N$ we define the subspace $O_{x}$ of $N_{x}$ by

$$
O_{x}=\operatorname{span}\left\{h(X, Y) \mid X, Y \in T_{x} M^{n}\right\},
$$

called the first affine normal space.

Lemma 1.1. The dimension of $O_{x}$ does not depend on the choice of the transversal bundle $N$.

Pr o of. Let $N, \bar{N}$ be two affine normal bundles, $h, \bar{h}$ the associated second fundamental forms and $O_{x}, \bar{O}_{x}$ the first affine normal spaces induced by $h$ and $\bar{h}$, respectively. For $x \in M^{n}$ let

$$
\pi_{x}: f_{*}\left(T_{x} M^{n}\right) \oplus \bar{N}_{x} \rightarrow \bar{N}_{x}
$$

be the projection. Then $\bar{\pi}_{x}=\left.\pi\right|_{N_{x}}$ is an isomorphism. For every $X, Y \in$ $T_{x} M^{n}$ we have

$$
\bar{\pi}_{x}(h(X, Y))=\pi_{x}\left(\widetilde{\nabla}_{X} f_{*}(Y)-f_{*}\left(\nabla_{X} Y\right)\right)=\pi_{x}\left(\widetilde{\nabla}_{X} f_{*}(Y)\right)=\bar{h}(X, Y) .
$$

This implies that $\left.\pi\right|_{O_{x}}$ is an isomorphism between $O_{x}$ and $\bar{O}_{x}$, which completes the proof.

We now adapt the notion of the type number of an immersion used in the Riemannian case to affine geometry. First we define the type number of a set of bilinear forms.

Let $V$ be an $n$-dimensional vector space and $h^{1}, \ldots, h^{k}$ be linearly independent bilinear forms on $V \times V$. We define mappings $\Phi^{i}: V \rightarrow V^{*}$ (for $i=1, \ldots, k)$ as follows:

$$
\Phi^{i}(v)(w)=h^{i}(v, w) .
$$

The type number of the set $\left\{h^{1}, \ldots, h^{k}\right\}$ is the maximal integer $r$ such that there exist $r$ vectors $v_{1}, \ldots, v_{r} \in V$ for which $\Phi^{i}\left(v_{j}\right)$ are linearly independent for $i=1, \ldots, k$ and $j=1, \ldots, r$. 
Now we define the type number of an immersion $f: M^{n} \rightarrow \mathbb{R}^{n+p}$ at $x \in M^{n}$ (compare [1], [3], [6]). Choose a basis $\left\{\xi_{1}, \ldots, \xi_{k}\right\}$ of $O_{x}$. Then

$$
h(X, Y)=\sum_{i=1}^{k} h^{i}(X, Y) \xi_{i}
$$

for every $X, Y \in T_{x} M^{n}$. It is clear that $h^{1}, \ldots, h^{k}: T_{x} M^{n} \times T_{x} M^{n} \rightarrow \mathbb{R}$ are symmetric bilinear forms.

Definition 1.2. The type number of an affine immersion $f$ at $x \in M^{n}$, denoted by $t_{x} f$, is the type number of the set $\left\{h^{1}, \ldots, h^{k}\right\}$ of symmetric bilinear forms defined above.

We prove that $t_{x} f$ is a well defined notion.

Lemma 1.3. The forms $h^{1}, \ldots, h^{k}$ are linearly independent.

Proof. Assume for contradiction that there exist numbers $\alpha_{1}, \ldots, \alpha_{k}$ such that

$$
h^{j}=\sum_{i \neq j} \alpha_{i} h^{i}
$$

for some $j \leq k$. Then

$$
h=\sum_{i=1}^{k} h^{i} \xi_{i}=\sum_{i \neq j} h^{i} \xi_{i}+\sum_{i \neq j} \alpha_{i} h^{i} \xi_{j}=\sum_{i \neq j} h^{i}\left(\xi_{i}+\alpha_{i} \xi_{j}\right)
$$

and now $O_{x}$ is spanned by the $k-1$ vectors $\xi_{i}+\alpha_{i} \xi_{j}$ for $i \in\{1, \ldots, k\} \backslash\{j\}$.

Lemma 1.4. $t_{x} f$ is independent of the choice of $N$ and $\left\{\xi_{1}, \ldots, \xi_{k}\right\}$.

Proof. Let $N_{x}$ and $\bar{N}_{x}$ be two transversal spaces and let $\xi_{1}, \ldots, \xi_{k}$ and $\bar{\xi}_{1}, \ldots, \bar{\xi}_{k}$ span $O_{x} \subset N_{x}$ and $\bar{O}_{x} \subset \bar{N}_{x}$ respectively. Let $\bar{\xi}_{1}, \ldots, \bar{\xi}_{k}, \bar{\xi}_{k+1}$, $\ldots, \bar{\xi}_{p}$ span the whole space $\bar{N}_{x}$. Then

$$
\xi_{j}=\sum_{i=1}^{p} a_{i j} \bar{\xi}_{i}+Z_{j}
$$

where $a_{i j} \in \mathbb{R}$ and $Z_{j} \in T_{x} M^{n}$. If $\nabla$ and $\bar{\nabla}$ are the connections on $M^{n}$ defined by $N$ and $\bar{N}$, then

$$
f_{*}\left(\nabla_{X} Y\right)+\sum_{j=1}^{k} h^{j}(X, Y) \xi_{j}=f_{*}\left(\bar{\nabla}_{X} Y\right)+\sum_{i=1}^{k} \bar{h}^{i}(X, Y) \bar{\xi}_{i}
$$

and 


$$
\begin{aligned}
\sum_{j=1}^{k} h^{j}(X, Y) Z_{j}+f_{*}\left(\nabla_{X} Y\right)+\sum_{i=1}^{p} \sum_{j=1}^{k} a_{i j} h^{j}(X, Y) \bar{\xi}_{i} & \\
& =f_{*}\left(\bar{\nabla}_{X} Y\right)+\sum_{i=1}^{k} \bar{h}^{i}(X, Y) \bar{\xi}_{i}
\end{aligned}
$$

for every $X, Y \in T_{x} M^{n}$. Hence $\bar{h}^{i}=\sum_{j=1}^{k} a_{i j} h^{j}$ for $i=1, \ldots, k$ and $\sum_{j=1}^{k} a_{i j} h^{j}=0$ for $i=k+1, \ldots, p$, which gives $a_{i j}=0$ for $i>k$ by the previous lemma. Thus the matrix $\left[a_{i j}\right]_{i, j=1, \ldots, k}$ is non-singular. Now the equation

$$
\bar{\Phi}^{i}=\sum_{j=1}^{k} a_{i j} \Phi^{j}
$$

implies that if $\Phi^{j}\left(X_{s}\right)$ are linearly independent for $s=1, \ldots, r$ and $j=$ $1, \ldots, k$ then so are $\bar{\Phi}^{j}\left(X_{s}\right)$. The proof is complete.

We now prove an algebraic lemma which will be useful later.

LEMMA 1.5. Let $V, W$ be vector spaces, and $h^{1}, \ldots, h^{k}$ be bilinear forms on $V \times V$ with type number greater than one. Let $B_{i}: V \rightarrow W$ be linear maps for $i=1, \ldots, k$. If

$$
\sum_{i=1}^{k}\left\{h^{i}(X, Z) B_{i}(Y)-h^{i}(Y, Z) B_{i}(X)\right\}=0
$$

for every $X, Y, Z \in V$, then $B_{i}=0$ for $i=1, \ldots, k$.

Proof. It is sufficient to assume that $W=\mathbb{R}$. The equation $(*)$ with fixed $X$ and $Y$ but arbitrary $Z$ means that

$$
\sum_{i=1}^{k}\left\{B_{i}(Y) \Phi^{i}(X)-B_{i}(X) \Phi^{i}(Y)\right\}=0,
$$

where $\Phi^{i}: V \rightarrow V^{*}$ is given by $\Phi^{i}(v)(w)=h^{i}(v, w)$. Because the type number of $\left\{h^{1}, \ldots, h^{k}\right\}$ is at least 2 , we can take $X, Y \in V$ such that the set $\left\{\Phi^{i}(X), \Phi^{i}(Y)\right\}_{i=1, \ldots, k}$ is linearly independent. But this immediately implies $B_{i}(X)=B_{i}(Y)=0$. Now we substitute in (**) an arbitrary vector $Z \in V$ in place of $Y$ and obtain

$$
\sum_{i=1}^{k} B_{i}(Z) \Phi^{i}(X)=0
$$

whence $B_{i}(Z)=0$ for every $Z \in V$ and $i=1, \ldots, k$. The proof is complete.

R e m a r k 1.6. The definition of the type number of an immersion generalizes the corresponding definitions in the Riemannian case and in the case of hypersurfaces in affine geometry $([3],[6],[5])$. 
Namely, if $\mathbb{R}^{n+p}$ is equipped with the standard scalar product $\langle$,$\rangle and$ $f: M^{n} \rightarrow \mathbb{R}^{n+p}$ is an immersion, we can take for $N$ in (1.1) the normal bundle and for $h$ in (1.2) the Riemannian second fundamental form with respect to $\langle$,$\rangle . Then \left\langle A_{\xi} X, Y\right\rangle=\langle h(X, Y), \xi\rangle$, where $X, Y \in T_{x} M^{n}$.

According to [6], the type number of $f$ is equal to the maximal integer $r$ such that there exist $r$ vectors $X_{1}, \ldots, X_{r} \in T_{x} M^{n}$ for which the set $\left\{A_{\xi_{i}} X_{j}\right.$ : $i=1, \ldots, k ; j=1, \ldots, r\}$ is linearly independent, where $\left(\xi_{1}, \ldots, \xi_{k}\right)$ is a local frame on $N$. Let $X_{1}, \ldots, X_{r} \in T_{x} M^{n}$, and let $\left(\xi_{1}, \ldots, \xi_{k}\right)$ be a local frame on $N$. Let $\Phi^{i}$ denote mappings as defined before Definition 1.2, and $a_{i}^{j}$ real numbers. We have $h^{i}(X, Y)=\left\langle h(X, Y), \xi_{i}\right\rangle$. Notice that the following conditions are equivalent:

$$
\begin{gathered}
\sum_{i, j} a_{i}^{j} \Phi^{i}\left(X_{j}\right)=0, \\
\forall Y \in T_{x} M^{n} \quad \sum_{i, j} a_{i}^{j} h^{i}\left(X_{j}, Y\right)=0, \\
\forall Y \in T_{x} M^{n} \quad \sum_{i, j}\left\langle a_{i}^{j} A_{\xi_{i}} X_{j}, Y\right\rangle=0, \\
\sum_{i, j} a_{i}^{j} A_{\xi_{i}} X_{j}=0 .
\end{gathered}
$$

This means that the set $\left\{A_{\xi_{i}} X_{j}: i=1, \ldots, k ; j=1, \ldots, r\right\}$ is linearly independent if and only if $\left\{\Phi^{i}\left(X_{j}\right): i=1, \ldots, k ; j=1, \ldots, r\right\}$ is. Thus the remark is true in the Riemannian case.

In the case of hypersurfaces the type number of $f$ is the rank of the second fundamental form, but the latter is equal to the rank of $\Phi$.

2. Basic equations. We recall the equations of affine geometry (see for example [2]).

Let $f: M^{n} \rightarrow \mathbb{R}^{n+p}$ be an immersion. If we have $N$ and an affine connection $\nabla$ on $M^{n}$ such that (1.1) and (1.2) are satisfied, we call such an immersion an affine immersion and we write

$$
f:\left(M^{n}, \nabla\right) \rightarrow\left(\mathbb{R}^{n+p}, \widetilde{\nabla}\right) .
$$

Denoting the curvature tensors of $\nabla$ and $D$ by $R$ and $R^{\perp}$ respectively, we have the following equations:

$$
\begin{aligned}
R(X, Y) Z & =A_{h(Y, Z)} X-A_{h(X, Z)} Y & & \text { (Gauss), } \\
\nabla h(X, Y, Z) & =\nabla h(Y, X, Z) & & \text { (Codazzi), } \\
R^{\perp}(X, Y) \xi & =h\left(X, A_{\xi} Y\right)-h\left(A_{\xi} X, Y\right) & & \text { (Ricci), }
\end{aligned}
$$

where $\nabla h(X, Y, Z)=D_{X} h(Y, Z)-h\left(\nabla_{X} Y, Z\right)-h\left(Y, \nabla_{X} Z\right)$ for every $X, Y, Z$ 
$\in \mathcal{X}\left(M^{n}\right)$, and $\xi \in \mathcal{X}(N)$. Here $\mathcal{X}(N)$ denotes the module of local sections of the transversal bundle $N$ over $M^{n}$.

3. Reduction of codimension. We use a result from [4]:

Proposition 3.1. Let $f:\left(M^{n}, \nabla\right) \rightarrow\left(\mathbb{R}^{n+p}, \widetilde{\nabla}\right)$ be an affine immersion. Suppose that $N_{1}$ is a subbundle of the normal bundle $N$ such that:

(1) $N_{1}$ contains $O_{x}$ for every $x \in M^{n}$.

(2) $N_{1}$ is parallel relative to the normal connection $D$.

Then $f\left(M^{n}\right)$ is contained in an $(n+q)$-dimensional affine subspace of $\mathbb{R}^{n+p}$, where $q=\operatorname{dim} N_{1}(x)$.

We now formulate a reduction lemma which involves the notion of the type number of an affine immersion. It is actually a generalization of Lemma 28 from [6] to the case when the ambient manifold is the affine space $\left(\mathbb{R}^{n+p}, \widetilde{\nabla}\right)$.

Proposition 3.2. Let $f:\left(M^{n}, \nabla\right) \rightarrow\left(\mathbb{R}^{n+p}, \widetilde{\nabla}\right)$ be an affine immersion such that:

(1) $t_{x} f \geq 2$ at every $x \in M^{n}$.

(2) $\operatorname{dim} O_{x}=k$ on $M^{n}$.

Then $f\left(M^{n}\right)$ is contained in an $(n+q)$-dimensional affine subspace of $\mathbb{R}^{n+p}$.

Proof. We prove that the subbundle $O$ of $N$ satisfies the assumptions of the previous proposition. For every $x \in M^{n}$ let $W_{x}$ be a vector subspace of $N_{x}$ such that $N_{x}=O_{x} \oplus W_{x}$. Let $\xi$ be a section of $O$ in an open neighbourhood of $x \in M^{n}$. We now prove that $\left(D_{X} \xi\right)(x) \in O_{x}$ for every $X \in \mathcal{X}\left(M^{n}\right)$.

Let $\xi_{1}, \ldots, \xi_{k}$ be sections of $N$ which span $O_{x}$ and $\xi_{k+1}, \ldots, \xi_{p}$ be sections which span $W_{x}$. Then there exists a neighbourhood $U_{x}$ of $x$ and functions $a_{1}, \ldots, a_{k}$ in $U_{x}$ such that $\xi=\sum_{i=1}^{k} a_{i} \xi_{i}$. We also have a decomposition

$$
\left(D_{X} \xi\right)(y)=\left(D_{X}^{1} \xi\right)(y)+\left(D_{X}^{2} \xi\right)(y),
$$

where $\left(D_{X}^{1} \xi\right)(y) \in O_{y}$ and $\left(D_{X}^{2} \xi\right)(y) \in W_{y}$ for $y \in U_{x}$. The mapping $X \mapsto\left(D_{X} \xi\right)(x)$ is obviously $\mathbb{R}$-linear and so is $X \mapsto\left(D_{X}^{2} \xi\right)(x)$. Notice that the second fundamental form is $h=\sum_{i=1}^{k} h^{i} \xi_{i}$ and consider the Codazzi equation for arbitrary $X, Y, Z \in \mathcal{X}\left(M^{n}\right)$. Comparing its components belonging to $W_{x}$ gives

$$
\sum_{i=1}^{k} h^{i}(Y, Z) D_{X}^{2} \xi_{i}=\sum_{i=1}^{k} h^{i}(X, Z) D_{Y}^{2} \xi_{i} .
$$


By Lemma 1.5 we have $\left(D_{X}^{2} \xi_{i}\right)(x)=0$ and therefore $\left(D_{X} \xi_{i}\right)(x) \in O_{x}$ for $i=1, \ldots, k$. Hence $\left(D_{X} \xi_{i}\right)(x)=\sum_{i=1}^{k} a_{i}(x)\left(D_{X} \xi_{i}\right)(x)+\sum_{i=1}^{k} X\left(a_{i}\right) \xi_{i}(x)$ $\in O_{x}$. An application of the above proposition completes the proof.

4. An equivalence theorem. Dillen's paper [2] contains a general equivalence theorem for immersed manifolds of any codimension. Under an assumption about the type number we can formulate a stronger result. First we recall the result of Dillen.

THEOREM 4.1. Let $f, \bar{f}:\left(M^{n}, \nabla\right) \rightarrow\left(\mathbb{R}^{n+p}, \widetilde{\nabla}\right)$ be affine immersions with corresponding affine normal spaces $N$ and $\bar{N}$, second fundamental forms $h$ and $\bar{h}$, affine shape operators $A$ and $\bar{A}$, and normal connections $D$ and $\bar{D}$, respectively. Suppose that there exists an isomorphism $F: N \rightarrow \bar{N}$ of vector bundles over $M^{n}$ such that:

(1) $F \circ h=\bar{h}$.

(2) $A_{\xi}=\bar{A}_{F(\xi)}$.

(3) $F\left(D_{X} \xi\right)=\bar{D}_{X} F(\xi)$, where $X \in \mathcal{X}\left(M^{n}\right)$ and $\xi \in \mathcal{X}(N)$.

Then there exists $B \in A(n+p, \mathbb{R})$ such that $\bar{f}=B \circ f$, where $A(n+p, \mathbb{R})$ denotes the group of affine transformations of $\mathbb{R}^{n+p}$.

The main result of this section is the following theorem.

Theorem 4.2. Let $f, \bar{f}:\left(M^{n}, \nabla\right) \rightarrow\left(\mathbb{R}^{n+p}, \widetilde{\nabla}\right)$ be affine immersions. Suppose that:

(1) $t_{x} f \geq 2$ at every point $x$ in $M^{n}$.

(2) There exists an isomorphism $F: N \rightarrow \bar{N}$ of vector bundles over $M^{n}$ such that $F \circ h=\bar{h}$.

(3) $\operatorname{dim} O_{x}=k$ for every $x \in M^{n}$.

Then there exists $B \in A(n+p, \mathbb{R})$ such that $\bar{f}=B \circ f$.

P r o o f. Proposition 3.2 allows us to consider only the case of $p=k$. By (2), we have $\operatorname{dim} O_{x}=\operatorname{dim} \bar{O}_{x}$. We take a set $\left\{\xi_{1}, \ldots, \xi_{k}\right\}$ of sections of $N$ that span $O_{x}$ in a certain neighbourhood of a fixed point of $M^{n}$.

Let $\left\{\bar{\xi}_{1}, \ldots, \bar{\xi}_{k}\right\}$ be the sections given by $F\left(\xi_{i}\right)=\bar{\xi}_{i}$ for $i=1, \ldots, k$. Then there exist two sets of symmetric bilinear forms on $T_{x} M^{n},\left\{h^{1}, \ldots, h^{k}\right\}$ and $\left\{\bar{h}^{1}, \ldots, \bar{h}^{k}\right\}$, such that $h=\sum h^{i} \xi_{i}$ and $\bar{h}=\sum \bar{h}^{i} \bar{\xi}_{i}$.

By the assumptions we have

$$
\sum \bar{h}^{i} \bar{\xi}_{i}=F\left(\sum h^{i} \xi_{i}\right)=\sum h^{i} \bar{\xi}_{i} .
$$

Hence $\bar{h}^{i}=h^{i}$ for $i=1, \ldots, k$.

The Gauss equations for $f$ and $\bar{f}$ imply that

$$
A_{h(Y, Z)}(X)-A_{h(X, Z)}(Y)=\bar{A}_{\bar{h}(Y, Z)}(X)-\bar{A}_{\bar{h}(X, Z)}(Y),
$$


whence

$$
\sum h^{i}(Y, Z)\left(A_{\xi_{i}}-\bar{A}_{\bar{\xi}_{i}}\right)(X)=\sum h^{i}(X, Z)\left(A_{\xi_{i}}-\bar{A}_{\bar{\xi}_{i}}\right)(Y) .
$$

Using Lemma 1.5 we obtain $A_{\xi_{i}}=\bar{A}_{\bar{\xi}_{i}}$ for $i=1, \ldots, k$. This also means that $A_{\xi}=\bar{A}_{F(\xi)}$ for every section $\xi$ of $N$. The condition (2) from the previous theorem is satisfied.

Now we apply $F$ to the Codazzi equation for $f$ and use the equality $F \circ h=\bar{h}$ :

$$
\begin{aligned}
0= & F(\nabla h(X, Y, Z)-\nabla h(Y, X, Z)) \\
= & F\left(D_{X} h(Y, Z)-D_{X} h(X, Z)\right) \\
& -\left(\bar{h}\left(\nabla_{X} Y, Z\right)+\bar{h}\left(Y, \nabla_{X} Z\right)-\bar{h}\left(\nabla_{Y} X, Z\right)-\bar{h}\left(X, \nabla_{Y} Z\right)\right) .
\end{aligned}
$$

Next we compare the above equality with the Codazzi equation for $\bar{f}$ :

$$
\begin{aligned}
0= & \bar{D}_{X} \bar{h}(Y, Z)-\bar{D}_{Y} \bar{h}(X, Z) \\
& -\left(\bar{h}\left(\nabla_{X} Y, Z\right)+\bar{h}\left(Y, \nabla_{X} Z\right)-\bar{h}\left(\nabla_{Y} X, Z\right)-\bar{h}\left(X, \nabla_{Y} Z\right)\right)
\end{aligned}
$$

to obtain

$$
\bar{D}_{X} \bar{h}(Y, Z)-\bar{D}_{Y} \bar{h}(X, Z)=F\left(D_{X} h(Y, Z)-D_{X} h(X, Z)\right) .
$$

Since $\bar{h}^{i}=h^{i}$ and $F\left(\xi_{i}\right)=\bar{\xi}_{i}$, by straightforward computation we get

$$
\sum h^{i}(Y, Z)\left[\bar{D}_{X} \bar{\xi}_{i}-F\left(D_{X} \xi_{i}\right)\right]=\sum h^{i}(X, Z)\left[\bar{D}_{Y} \bar{\xi}_{i}-F\left(D_{Y} \xi_{i}\right)\right] .
$$

But the mappings $X \mapsto \bar{D}_{X} \bar{\xi}_{i}-F\left(D_{X} \xi_{i}\right)$ are linear for $i=1, \ldots, k$. Therefore from Lemma 1.5 we have $F\left(D_{X} \xi_{i}\right)=\bar{D}_{X} \bar{\xi}_{i}$ for $i=1, \ldots, k$, which also implies $F\left(D_{X} \xi\right)=\bar{D}_{X} \bar{\xi}$ for every section $\xi$ of $N$. By Theorem 4.1, this completes the proof.

Remark 4.3. The affine transformation $B$ obtained in Theorem 4.2 is unique (comp. [6]). Since $O_{x}=N_{x}$, it is enough to prove that each vector $X \in O_{x}$ is of the form $(f \circ \gamma)^{\prime \prime}(0)$ for a curve $\gamma$ on $M^{n}$. It is clear that the space $O_{x}$ is spanned by vectors of the form $h(X, X)$, where $X \in T_{x} M^{n}$. If we take a geodesic $\gamma$ on $M^{n}$ such that $\gamma^{\prime}(0)=X$, then

$$
(f \circ \gamma)^{\prime \prime}(0)=\widetilde{\nabla}_{\gamma}(f \circ \gamma)^{\prime}(0)=h\left(\gamma^{\prime}(0), \gamma^{\prime}(0)\right) .
$$

\section{References}

[1] C. B. Allendoerfer, Rigidity for spaces of class greater than one, Amer. J. Math. 61 (1939), 633-644.

[2] F. Dillen, Equivalence theorems in affine differential geometry, Geom. Dedicata 32 (1988), 81-92.

[3] S. Kobayashi and K. Nomizu, Foundations of Differential Geometry, Vol. II (Appendix), Wiley, New York, 1969. 
[4] K. Nomizu and U. Pinkall, Cubic form theorem for affine immersions, Results in Math. 13 (1988), 338-362.

[5] B. Opozda, Some equivalence theorems in affine hypersurface theory, Monatsh. Math. 113 (1992), 245-254.

[6] M. Spivak, A Copmprehensive Introduction to Differential Geometry, Vol. 5, Publish or Perish, 1979, 361-369.

INSTITUTE OF MATHEMATICS

PEDAGOGICAL UNIVERSITY

W. POLA 2

35-959 RZESZÓW, POLAND

Reçu par la Rédaction le 5.7.1993

Révisé le 8.4.1994 